\title{
高比能量锂离子电池硅基负极材料研究进展
}

\author{
谭 毅 ${ }^{1,2}$, 王 凯 ${ }^{1,2}$
}

(大连理工大学 1. 材料科学与工程学院; 2. 辽宁省太阳能光伏系统重点实验室, 大连 116024)

摘 要: 硅的理论嵌锂比容量是石墨材料比容量的十倍以上, 脱锂电位低, 资源丰富, 倍率特性较好, 故高比能量 的硅基材料成为了电动汽车、可再生能源储能系统等领域的研究热点。但由于其在脱嵌锂过程中巨大的体积膨胀效 应会导致硅电极材料粉化和结构崩塌, 并且在电解液中硅表面重复形成的固相电解质层(SEI)使极化增大、库伦效 率降低, 最终导致电化学性能的恶化。为了解决上述问题, 加快实现硅基电极的商业化应用, 本文系统总结了通过 硅基材料的选择和结构设计来解决充放电过程中体积效应的工作, 并深入分析和讨论了具有代表性的硅基复合材 料的制备方法、电化学性能和相应机理, 重点介绍了硅碳复合材料和 $\mathrm{SiO}_{x}(0<x \leqslant 2)$ 基复合材料。最后对硅基负极材 料存在的问题进行了分析, 并展望了其研究前景。

关 键 词: 硅基材料; 负极材料; 锂离子电池; 综述

中图分类号: TM912 文献标识码: A

\section{Silicon-based Anode Materials Applied in High Specific Energy Lithium-ion Batteries: a Review}

\begin{abstract}
TAN Yi ${ }^{1,2}$, WANG Kai ${ }^{1,2}$
(1. School of Materials Science and Engineering, Dalian University of Technology, Dalian 116024, China; 2. Key Laboratory for Solar Energy Photovoltaic System of Liaoning Province, Dalian 116024, China)

Abstract: Silicon has the highest theoretical lithium insertion specific capacity, more than ten times the theoretical specific capacity of graphite electrode material, and low delithiation potential, with abundant resources and good rate characteristics, high-energy-density lithium-ion battery silicon-based materials have become hot spots in application fields such as electric vehicles and renewable energy storage systems. However, it will cause powdering and structural collapse of the silicon electrode material due to its large volume expansion effect in the process of delithiation and lithium insertion. In addition, the solid electrolyte interface (SEI) layer on the surface of silicon is repeatedly formed in the electrolyte, which increases the polarization and reduces the coulomb efficiency, eventually leading to deterioration of electrochemical performance. In order to solve the above problems and realize the commercial application of silicon electrodes. This paper systematically summarizes the work to solve the volume effect in charge and discharge process through the selection and structural design of silicon-based materials, and deeply analyzes and discusses the preparation methods, electrochemical properties and corresponding mechanisms of representative silicon-based composite materials, focusing on silicon-carbon composites and $\mathrm{SiO}_{x}(0<x \leqslant 2)$ based anode materials. Finally, the problems of silicon-based anode materials are analyzed and their prospects are prospected.
\end{abstract}

Key words: silicon-based material; anode materials; lithium-ion batteries; review 
锂离子电池由于脱锂电位低, 资源丰富, 绿色 环保, 比能量较高、无记忆效应和工作电压高等优 势，在手机、笔记本电脑及数码相机等电子产品领域 得到了广泛应用。高比能量的锂离子电池从电子终 端设备走向电动汽车和储能技术领域势在必行 ${ }^{[1-2]}$ 。 常见的锂离子电池负极材料有软碳、硬碳、中间相 碳微球(MCMB)、人造石墨、天然石墨、钛酸锂(LTO) 和硅基材料等。目前, 锂离子电池商用负极材料石 墨的比容量已接近理论值 $(372 \mathrm{mAh} / \mathrm{g})$, 很难再有质 的提升, LTO 虽然循环安全性较好, 但是比容量太 低(176 mAh/g), 难以满足未来高比能量电池的发展 需求。由几种负极材料的性能对比图(图 1)可见, 要 满足 2020 年高能量密度锂离子二次电池 $300 \mathrm{Wh} / \mathrm{kg}$ 以上的需求, 高容量低成本低电压平台的硅基材料 是具有极大的潜力。

锂离子嵌入过程中形成硅锂合金 $\mathrm{Li}_{22} \mathrm{Si}_{5}$ 相, 对 应的理论容量是天然石墨的十多倍。同时, 硅在地 球上储量丰富, 生产成本很低, 且硅的电压平台为 0.3 0.5 V, 在充电过程中不存在析锂隐患, 大大提 高了锂离子电池的安全使用性能。但其在充放电过程 中，由于锂化和脱锂循环期间的高体积变化 $(200 \%$ $300 \%)$, 造成颗粒粉碎和其表面的固相电解质层 (SEI) 重复形成, 最终导致硅基负极材料容量的损耗 和循环性能较差等问题 ${ }^{[3-4]}$ 。

针对硅基负极材料在嵌锂和脱锂中存在的问题, 近些年研究者们通过对硅基材料的纳米化、复合化 和核壳结构的改性设计, 有效地提高了硅基材料的 循环性能。本文主要深入分析和讨论硅基负极材料 的储能及容量衰减机理, 从硅碳复合材料和 $\mathrm{SiO}_{x}$ $(0<x \leqslant 2)$ 基复合材料两条主线出发详细总结了硅基 电极材料的结构设计及改性研究的最新国内外进展, 对未来的研究方向和应用前景作出了展望。

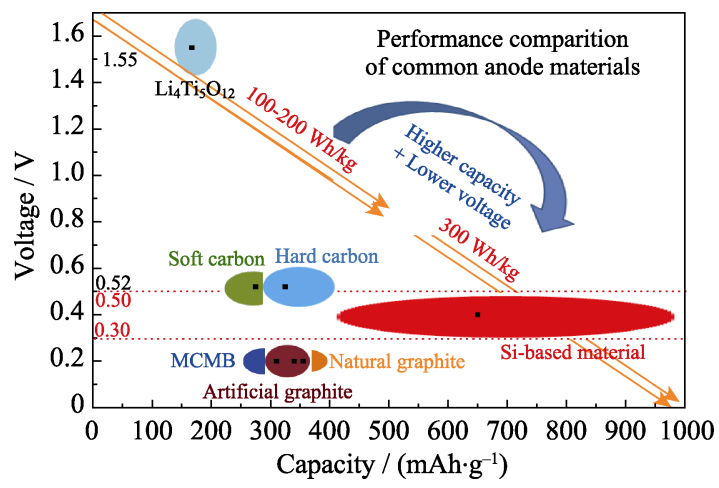

图 1 几种负极材料性能对比

Fig. 1 Performance comparison of common anode materials

\section{1 硅的电化学机理}

\section{1 脱嵌锂机理}

硅的脱嵌锂机理是通过与锂离子的合金化和 去合金化进行的。实际电化学嵌锂是晶态硅与非晶 亚稳态 $\mathrm{Li}_{x} \mathrm{Si}$ 共存的过程 ${ }^{[5-7]}$ 。研究发现, 在低充电 电位 $<0.5 \mathrm{~V}$ (相对于 $\mathrm{Li} / \mathrm{Li}^{+}$) 时, 硅锂合金化后最终形 成相常见的是 $\mathrm{Li}_{15} \mathrm{Si}_{4}$, 对应的理论质量比容量为 $3579 \mathrm{mAh} / \mathrm{g}^{[8]}$ 。硅在常温下充放电过程如下式(1) (3) 所示: 式(1)和(2)表示嵌锂过程; 式(3)表示脱锂过程; 式中 a 代表无定型, c 代表结晶态。

$$
\begin{gathered}
\mathrm{Si}(\mathrm{c})+x \mathrm{Li}^{+}+x \mathrm{e}^{-} \rightarrow \mathrm{Li}_{x} \mathrm{Si}(\mathrm{a}) \\
\mathrm{Li}_{x} \mathrm{Si}(\mathrm{a})+(3.75-x) \mathrm{Li}^{+}+(3.75-x) \mathrm{e}^{-} \rightarrow \mathrm{Li}_{15} \mathrm{Si}_{4}(\mathrm{c}) \\
\mathrm{Li}_{15} \mathrm{Si}_{4}(\mathrm{c}) \rightarrow \mathrm{Si}(\mathrm{a})+y \mathrm{Li}^{+}+y \mathrm{e}^{-}+\mathrm{Li}_{15} \mathrm{Si}_{4}
\end{gathered}
$$

\section{2 失效机制}

硅在充放电过程中会发生巨大的体积膨胀效应, 如图 2 晶胞示意图所示, 理论上嵌锂生成 $\mathrm{Li}_{22} \mathrm{Si}_{5}$ 晶 胞体积膨胀 $300 \%$ 左右 ${ }^{[9-12]}$ 。嵌锂过程中, 电解质会 发生分解并沉积在硅表面形成 SEI 膜 ${ }^{[13-15]}$ 。其失效 机制如图 3 所示, 由于脱嵌锂过程中硅体积的持续 变化, 暴露在电解液中新的硅表面 SEI 膜厚度持续 增加, 最终导致界面阻抗升高。研究表明, 亚稳态硅 锂合金及硅与电解液发生化学反应，造成锂离子的 消耗, 增加了锂离子的扩散距离, 阻碍锂离子的顺 利脱嵌，最终造成硅基负极材料容量的损耗 ${ }^{[16]}$ 。

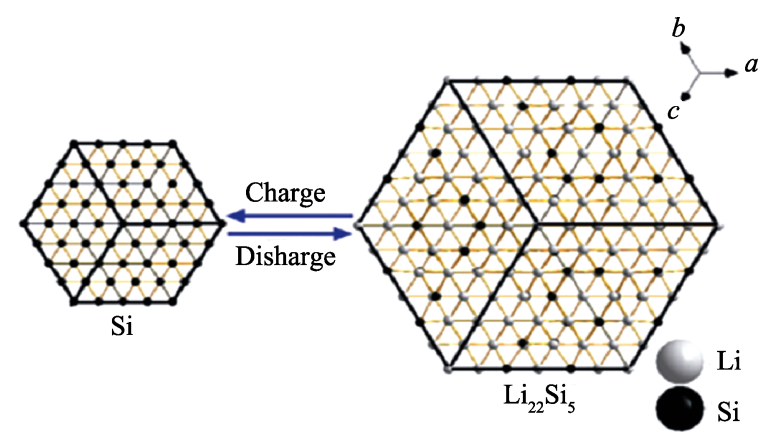

图 2 充电和放电期间硅体积膨胀示意图 ${ }^{[9]}$

Fig. 2 Illustration of Si volume expansion during charge and discharge ${ }^{[9]}$

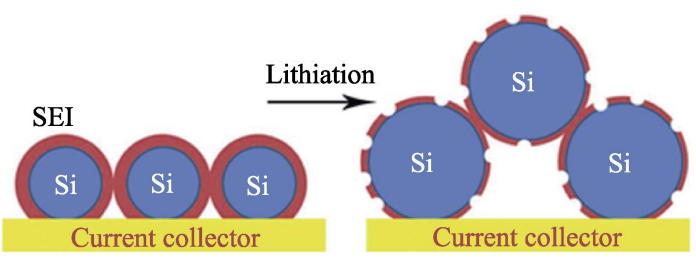

图 3 硅的失效机制示意图 ${ }^{[13]}$

Fig. 3 Schematic of the failure mechanism of silicon ${ }^{[13]}$ 


\section{2 硅基材料选择及设计}

\section{1 硅碳复合材料}

\subsection{1 低维化纳米硅碳复合材料}

研究者倾向对硅颗粒纳米化处理, 其具有较好的 电化学循环性能。但当硅颗粒尺寸小于 $100 \mathrm{~nm}$ 时, 在充放电过程中大的比表面积会与电解液发生更多 的接触, 形成更多的 SEI 膜, 且细小的纳米颗粒容易 发生团聚而加快容量的衰减, 故通常引入碳材料, 设 计出纳米硅管、薄膜等低维化的硅碳复合材料 ${ }^{[17-19]}$ 。 Kim 等 ${ }^{[20]}$ 通过镁热还原法制备的 CNT@mp-Si 纳米多 孔硅管的电极放电容量 300 次循环后为 $1019 \mathrm{mAh} / \mathrm{g}$, 对应的库仑效率为 99.6\%(见图 4)。可见硅纳米管较 大的空间有效改善了体积膨胀，材料具有优异的循 环性能。此外, 二维化的硅薄膜能够很好地缓冲硅 原子体积膨胀, 减少机械应力的产生, 进而提高硅 基材料的循环稳定性 ${ }^{[21-24]}$ 。Tong 等 ${ }^{[24]}$ 通过磁溅射方 法合成了非晶硅/碳 $(\mathrm{a}-\mathrm{Si} / \mathrm{C})$ 多层薄膜, 制备的微米级 $\mathrm{a}-\mathrm{Si} / \mathrm{C}$ 多层薄膜 $(1.1 \mathrm{~mm})$ 表现出良好的循环性能, 超过 200 个周期容量为 $1900 \mathrm{mAh} / \mathrm{g}$ 。

\subsection{2 三维化核壳硅碳复合材料}

硅基材料的核壳结构是通过系列设计方法将硅 与其它材料包覆起来而形成的特殊结构, 多孔结构 有利于锂离子快速传输, 且能有效缓冲循环过程中 的体积膨胀 ${ }^{[25-33]}$ 。多孔硅结构主要分为实心核壳结 构和中空核壳结构两大类。

\subsubsection{1 实心核壳硅碳材料}

Cui Yi 团队 ${ }^{[25]}$ 设计了一种石榴状的纳米硅碳材 料(图 5), 首先实心纳米硅颗粒外层包覆了有膨胀 空间的碳层, 然后多个颗粒复合组装, 在其表面再 包覆一层碳层, 最终形成微米级的实心核壳硅碳颗 粒, 且经过循环后颗粒表面形成稳定的 SEI 膜。其 在 1000 次循环后保持 $97 \%$ 的容量, 库伦效率达到 $99.87 \%$, 比容量可达 $1160 \mathrm{mAh} / \mathrm{g}$ 。Xie 等 ${ }^{[26]}$ 设计出 一种新型的实心蛋黄壳 $\mathrm{Si} @ \mathrm{C} @$ void@C 纳米复合 颗粒(图 6)。与 Si@void@C 材料相比, 所得的 $\mathrm{Si} @ \mathrm{C} @$ void@C 结构引入 $\mathrm{Si} @ \mathrm{C}$ 颗粒, 而不是像蛋 黄一样切断。这种额外的内部碳壳结构可以为 $\mathrm{Si}$ 核之间提供更好的电子传输和外部碳壳, 具有更小 的电荷转移阻抗。同时, 内外碳壳共同作用, 有效地 完全覆盖实心 $\mathrm{Si}$ 颗粒, 从而防止电极材料与电解质 直接接触而发生不可逆反应。Si@C@void@C 的初 始充电容量高达 $1910 \mathrm{mAh} / \mathrm{g}$, 并在 50 个周期后保 持 $71 \%$ 的容量。

\subsubsection{2 中空核壳硅碳材料}

虽然实心核壳结构能够改善硅材料的电化学性
能，但随着循环次数的增多，实心硅颗粒仍会面临 体积膨胀问题, 包覆表面的材料往往会产生裂纹从 而丧失特有的结构功能。因此若活性硅材料本身具 有一定的空隙结构, 可以为充放电过程中的体积膨 胀提供首要缓冲空间, 从而进一步提高材料的循环 性能 ${ }^{[27-29]}$ 。Bang 等 ${ }^{[27]}$ 利用 $\mathrm{Ag}$ 作为金属催化剂, 在 $\mathrm{HF}$ 和 $\mathrm{H}_{2} \mathrm{O}_{2}$ 的混合液中诱导腐蚀微米硅颗粒, 得到 多孔硅颗粒(图 7), 对其包碳处理后的核壳复合材 料, 首次比容量达到 $2050 \mathrm{mAh} / \mathrm{g}, 50$ 个周期后的容 量保持率为 $87 \%$ 。 $\mathrm{Ge}$ 等 ${ }^{[28-29]}$ 利用 $\mathrm{Ag}$ 作为金属催化 剂处理得到具有多孔结构的纳米硅, 再用 CVD 法 制备得到多孔硅/碳复合材料，其在 $0.5 C$ 下，200 个 周期后仍有 $945 \mathrm{mAh} / \mathrm{g}$ 的可逆比容量。

Tian 等 ${ }^{[30]}$ 将大量低成本 $\mathrm{Al}-\mathrm{Si}$ 合金铸锭通过酸 蚀刻，球磨和碳化处理合成了由 $20 \mathrm{~nm}$ 碳涂覆的二 次 $\mathrm{Si}$ 组成的微米尺寸 $\mathrm{Si} / \mathrm{C}$ 复合材料(图 8)。纳米多 孔 $\mathrm{Si} / \mathrm{C}$ 复合材料在电流密度为 $50 \mathrm{~mA} / \mathrm{g}$ 时，初始容

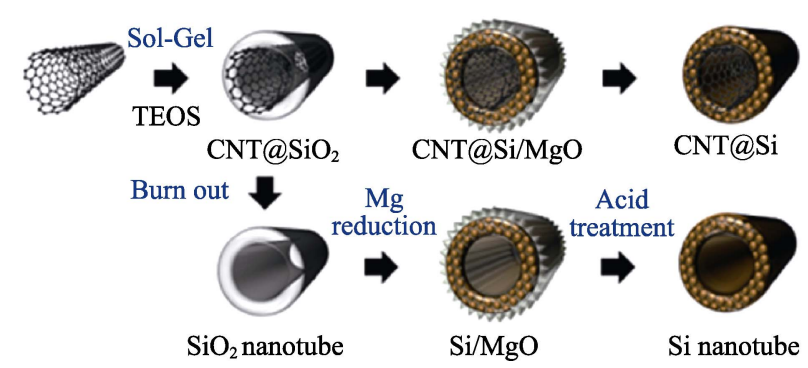

图 $4 \mathrm{CNT} @ \mathrm{mp}-\mathrm{Si}$ 和中孔多孔 $\mathrm{Si}$ 纳米管的合成示意图 ${ }^{[20]}$ Fig. 4 The composite map of synthesis of CNT@mp-Si and meso-porous Si nanotube ${ }^{[20]}$

(a)
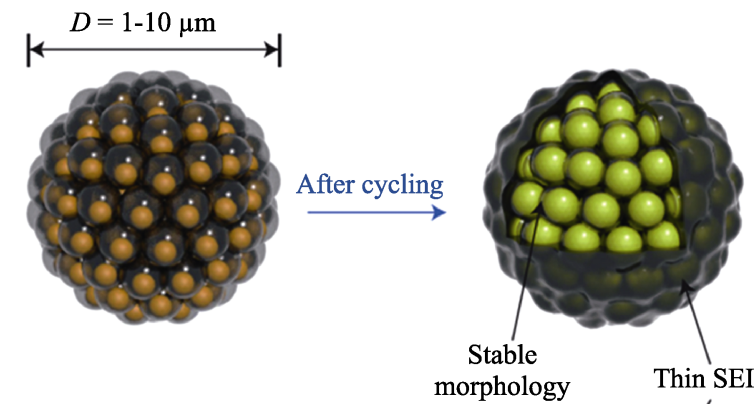

(b)

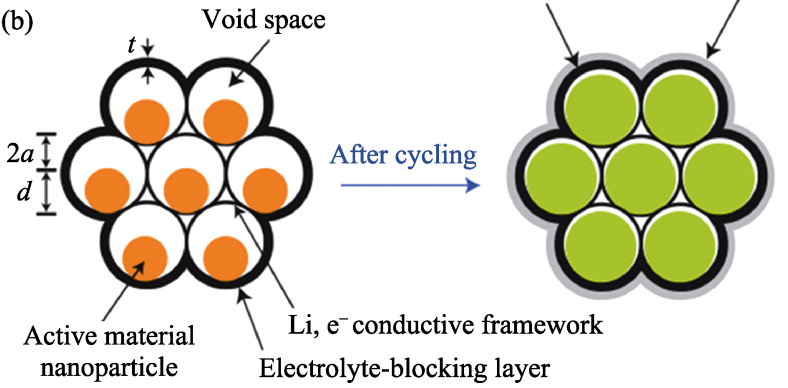

图 5 石榴状纳米硅碳材料设计示意图 ${ }^{[25]}$

Fig. 5 Schematic of the pomegranate-inspired design ${ }^{[25]}$ (a) Three dimensional view and (b) simplified two-dimensional cross-section view 
(a)

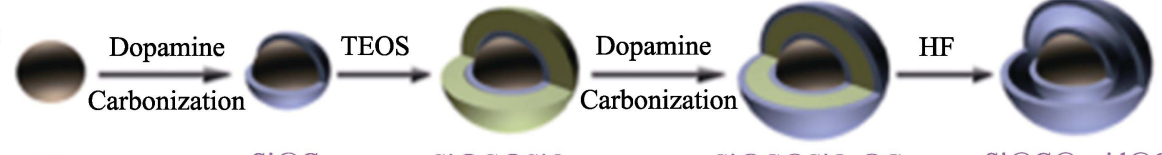

Si

Si@C@ $\mathrm{SiO}_{2}$

Si@C@SiO_@C

Si@C@void@C
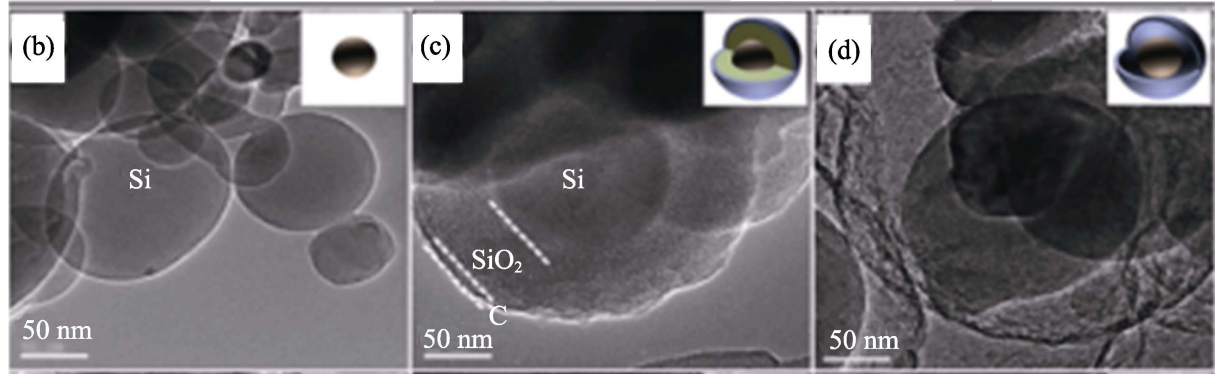

(e)
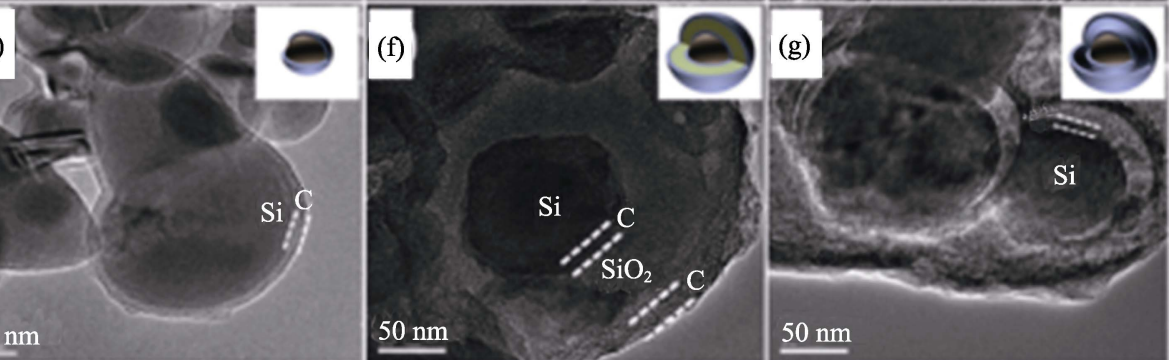

图 6 (a)新型实心核壳 $\mathrm{Si} @ \mathrm{C} @$ void@C 的形成过程示意图, (b)原料 $\mathrm{Si} 、$

(c)Si@SiO $\mathrm{SiC}_{2} @$ (d) Si@void@C、(e) Si@C、(f) Si@C@SiO $\mathrm{S}_{2} @ \mathrm{C}$ 和(g) Si@C@void@C 的 TEM 照片 ${ }^{[26]}$

Fig.6 (a) Schematic diagram of the novel core-shell Si@C@void@C, TEM images of (b) raw Si,

(c)Si@SiO $\mathrm{Si}_{2} @$, (d)Si@void@C, (e)Si@C, (f)Si@C@SiO $@$ C, and (g)Si@C@void@C ${ }^{[26]}$

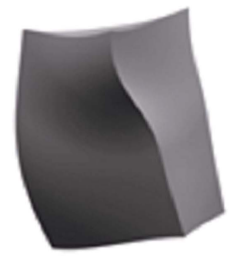

Bulk Si

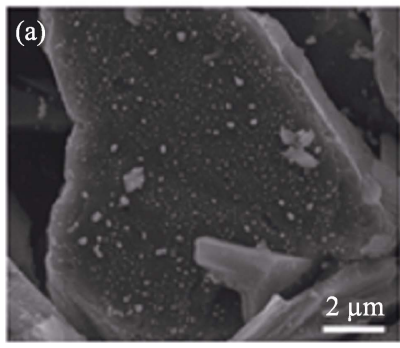

图 7 (a)硅表面沉积银颗粒和 $(b, c)$ 金属诱导刻蚀硅的扫描电镜照片 ${ }^{[28]}$

Fig. 7 Scanning diagrams of (a) Ag-deposited on $\mathrm{Si}$ and (b, c) metal induced etching $\mathrm{Si}^{[28]}$
Al-Si alloy

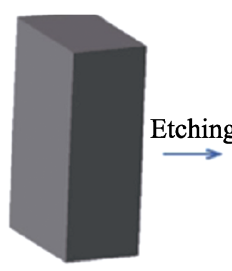

Porous Si
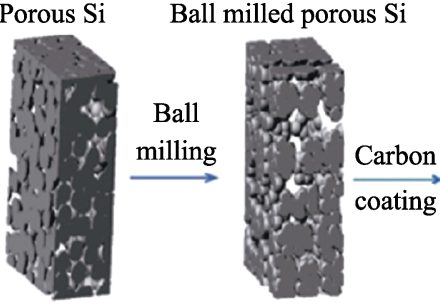

$\mathrm{Si} / \mathrm{C}$

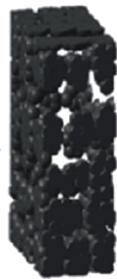

图 8 从 $\mathrm{Al}-\mathrm{Si}$ 合金到 $\mathrm{Si} / \mathrm{C}$ 复合材料的制备过程示意图 ${ }^{[30]}$

Fig. 8 Schematic illustration of the preparation process from Al-Si alloy to the $\mathrm{Si} / \mathrm{C}$ composite ${ }^{[30]}$
量为 $1182 \mathrm{mAh} / \mathrm{g}, 300$ 个循环后保持 $86.8 \%$ 的容量。

综上所述，在硅材料不同维度化结构设计中， 碳是最常用来与硅基材料复合。碳与硅复合能够降 低材料整体体积膨胀，同时起到抑制活性物质颗粒 团聚的作用, 此外碳的电导率较高, 与常规电解液 相容性好, 复合材料一般具有良好的循环稳定性能 和优异的导电性 ${ }^{[34]}$ 。碳材料可分为传统和新型两类, 其中传统碳材料主要包括软碳、硬碳、中间相碳微 球、人造石墨、天然石墨、炭黑等, 新型碳材料主 
要有碳纳米管/线和石墨烯等。目前, 新型碳材料在 学术科研上被广泛关注, 但相比新型碳材料的使用, 传统热解碳材料的成本低，适于商业化应用推广。

本课题组从清洁能源生产和废料回收利用大背 景下出发, 采用光伏产业中金刚线切割的微米硅粉为 原料, 通过系列预处理，将硅粉纯度从 $2 \mathrm{~N}(99 \%)$ 提纯 到 $5 \mathrm{~N}$ (99.999\%)左右，再利用高温热解碳制备的硅 碳负极材料循环 450 次后仍有 $426 \mathrm{mAh} / \mathrm{g}$ 以上的比 容量, 此工作对光伏产业金刚线切割废料的回收用 于锂离子电池负极材料具有重要的指导意义。表 1 系统总结了几种典型碳与硅复合负极材料的情况。

\section{$2.2 \mathrm{SiO}_{x}(0<x \leqslant 2) /$ 碳复合材料}

\subsection{1 $\mathrm{SiO}_{x}$ 材料的电化学机理}

$\mathrm{SiO}_{x}$ 材料主要由 $\mathrm{Si}$ 与 $\mathrm{SiO}_{2}$ 或者 $\mathrm{Si}$ 和 $\mathrm{O}_{2}$ 在高温 高真空下或惰性气氛下蒸镀制得(简称气相法 $)^{[40-41]}$, 本课题组采用 $\mathrm{Si}$ 与 $\mathrm{SiO}_{2}$ 在高温高真空条件下电子 束蒸镀得到 $\mathrm{SiO}_{x}$ 材料, 开展了部分 $\mathrm{SiO}_{x}$ 负极材料结 构改性的工作。 $\mathrm{SiO}_{x}$ 是一种结构相对复杂的非晶材 料, ICM (Interface Clusters Mixture) 模型认为, $\mathrm{SiO}_{x}$ 是由纳米 $\mathrm{Si}$ 、团簇分布 $\mathrm{SiO}_{2}$ 及亚氧化硅界面构成, 亚氧化界面区域占整体体积的比例介于 $20 \%$ 到 $25 \%$ 之间 ${ }^{[2-43]}$ 。 $\mathrm{SiO}_{x}$ 材料的电化学机理与单质 $\mathrm{Si}$ 有所区 别, 首先 $\mathrm{SiO}_{x}$ 与锂离子反应形成纳米 $\mathrm{Si} 、 \mathrm{Li}_{2} \mathrm{O}$ 及锂 硅酸盐, 生成的 $\mathrm{Li}_{2} \mathrm{O}$ 及锂硅酸盐主要抑制纳米 $\mathrm{Si}$ 颗粒发生团聚, 起到缓冲体积膨胀的作用 ${ }^{[44-45]}$ 。如 图 9 所示, Phillipe 等 ${ }^{[46]}$ 通过硬质和软质 XPS 研究了 硅纳米粒子和 $\mathrm{SiO}_{2}$ 层的界面机制, 类似于不成比例 的 $\mathrm{SiO}_{x}$ 纳米结构, 有助于理解 SEI 膜形成机制。其 中, $\mathrm{SiO}_{x}$ 材料的充放电机理一般可表示如下:

$$
\mathrm{SiO}_{x}+2 x \mathrm{Li} \rightarrow x \mathrm{Li}_{2} \mathrm{O}+\mathrm{Si}
$$

$$
\begin{gathered}
\mathrm{SiO}_{x}+x \mathrm{Li} \rightarrow 0.25 x \mathrm{Li}_{4} \mathrm{SiO}_{4}+(1-0.25 x) \mathrm{Si} \\
\mathrm{SiO}_{x}+0.4 x \mathrm{Li} \leftrightarrow 0.2 x \mathrm{Li}_{2} \mathrm{Si}_{2} \mathrm{O}_{5}+(1-0.4 x) \mathrm{Si} \\
\mathrm{Si}+3.75 \mathrm{Li} \leftrightarrow \mathrm{Li}_{3.75} \mathrm{Si}
\end{gathered}
$$

\subsection{2 $\mathrm{SiO}_{x} /$ 碳复合材料改性}

$\mathrm{SiO}_{x}$ 循环稳定性较单质 $\mathrm{Si}$ 有所提高, 但 $\mathrm{SiO}_{x}$ 材料在脱锂和嵌锂过程中仍然存在首次库伦效率 较低和体积膨胀较大的劣势。目前, 国内外研究者 采用歧化反应、复合化、预锂化和多孔化等手段对 $\mathrm{SiO}_{x}$ 基负极材料进行改性研究, 对提高材料的循 环性能、首次库伦效率和倍率性能具有一定的改善 效果。

\subsubsection{1 歧化处理}

利用 $\mathrm{SiO}_{x}$ 歧化反应改变材料内部的结构, 提高 循环性能。Park 等 ${ }^{[47]}$ 研究表明通过 $1000{ }^{\circ} \mathrm{C} 、 3 \mathrm{~h}$ 的 歧化反应和 $6 \mathrm{~h}$ 的高能球磨处理，制备得到的 nano-Si/ $\mathrm{SiO}_{x} /$ graphite 复合材料具有 $1516 \mathrm{mAh} / \mathrm{g}$ 的 初始放电容量, 100 个周期后容量保持率在 $70 \%$ 以 上，其循环性能明显优于未歧化热处理的 milled-SiO/graphite 材料。Morita 等 ${ }^{[48]}$ 通过歧化反应 和高温聚合反应制备得到纳米 $\mathrm{Si} / \mathrm{SiO}_{x} / \mathrm{C}$ 复合材料, 研究发现歧化反应后，更多的纳米 $\mathrm{Si}$ 颗粒或团簇颗 粒均匀分散在 $\mathrm{SiO}_{x}$ 基质中, 其 200 个周期后的比容 量仍有 $700 \mathrm{mAh} / \mathrm{g}$, 分析认为循环性能的提高归因 于纳米 $\mathrm{Si}$ 团簇颗粒在硅的氧化物中的高度分散性。

\subsubsection{2 预锂化处理}

预锂化处理提高材料的首次库伦效率。Yang 等 ${ }^{[49]}$ 研究发现在 $\mathrm{SiO} / \mathrm{C}$ 复合材料中混合一定量的金属锂 粉, 通过机械球磨和化学还原法制备得到纳米硅基 复合材料, 其初始容量达到 $770 \mathrm{mAh} / \mathrm{g}$, 首次库伦

\begin{tabular}{|c|c|c|c|c|c|}
\hline Composite type & Si source & Carbon source & Electrochemical performance & Method & Ref. \\
\hline $\mathrm{Si} /$ Porous-C & $\begin{array}{l}\text { Nano-silicon } \\
\text { powder }\end{array}$ & Pitch & $\begin{array}{c}723.8 \mathrm{mAh} / \mathrm{g}(1 \mathrm{st}) \\
600 \mathrm{mAh} / \mathrm{g}(100 \mathrm{~mA} / \mathrm{g}, 100)^{\mathrm{a}}\end{array}$ & $\begin{array}{c}\text { Spray drying }+ \\
\text { High-temperature pyrolysis }\end{array}$ & {$[35]$} \\
\hline Si@C@RGO & $\begin{array}{l}\text { Silicon powder } \\
\quad(80 \mathrm{~nm})\end{array}$ & Sucrose & $\begin{array}{c}1599 \mathrm{mAh} / \mathrm{g}(1 \mathrm{st}) \\
1517 \mathrm{mAh} / \mathrm{g}(100 \mathrm{~mA} / \mathrm{g}, 100)\end{array}$ & $\begin{array}{c}\text { Spray drying }+ \\
\text { High-temperature pyrolysis }\end{array}$ & {$[36]$} \\
\hline $\mathrm{Si} / \mathrm{C} / \mathrm{G}$ & $\begin{array}{l}\text { Silicon powder } \\
\quad(325 \text { mesh })\end{array}$ & $\begin{array}{l}\text { Phenol-formaldehyde } \\
\text { resin (PFR) }\end{array}$ & $\begin{array}{c}700 \mathrm{mAh} / \mathrm{g}(1 \mathrm{st}) \\
550 \mathrm{mAh} / \mathrm{g}(100 \mathrm{~mA} / \mathrm{g}, 40)\end{array}$ & High-temperature pyrolysis & {$[37]$} \\
\hline Silicon-sponge & $\begin{array}{l}\text { Si wafer } \\
(>20 \mu \mathrm{m})\end{array}$ & Acetylene & $\begin{array}{c}790 \mathrm{mAh} / \mathrm{g}(1 \mathrm{st}) \\
726 \mathrm{mAh} / \mathrm{g}(100 \mathrm{~mA} / \mathrm{g}, 300)\end{array}$ & $\begin{array}{l}\text { Electrochemical etching+ } \\
\text { High-temperature pyrolysis }\end{array}$ & {$[38]$} \\
\hline $\mathrm{PS} @ \mathrm{C}$ & $\begin{array}{l}\text { Si powder } \\
\quad(5 \mu \mathrm{m})\end{array}$ & Propylene & $\begin{array}{c}1980 \mathrm{mAh} / \mathrm{g}(1 \mathrm{st}) \\
1287 \mathrm{mAh} / \mathrm{g}(100 \mathrm{~mA} / \mathrm{g}, 100)\end{array}$ & Chemical etching + CVD & [39] \\
\hline $\mathrm{Si} / \mathrm{C}$ & $\begin{array}{l}\text { Al-Si alloy } \\
(2-10 \mu \mathrm{m})\end{array}$ & Polyacrylonitrile (PAN) & $\begin{array}{c}952 \mathrm{mAh} / \mathrm{g}(1 \mathrm{st}) \\
826.3 \mathrm{mAh} / \mathrm{g}(200 \mathrm{~mA} / \mathrm{g}, 300)\end{array}$ & $\begin{array}{l}\text { Chemical etching }+ \\
\text { High-temperature pyrolysis }\end{array}$ & {$[30]$} \\
\hline
\end{tabular}
效率达到 $81 \%$ 。Seong 等 ${ }^{[50]}$ 通过在 $\mathrm{SiO} / \mathrm{C}$ 电极表面

表 1 硅/碳锂离子电池负极材料的电化学性能

Table 1 Electrochemical performance of some silicon/carbon composite anodes for lithium-ion batteries

Note: ${ }^{-}$" $723.8 \mathrm{mAh} / \mathrm{g}(1 \mathrm{st}), 600 \mathrm{mAh} / \mathrm{g}(100 \mathrm{~mA} / \mathrm{g}, 100)$ " indicates that 1 th cycle discharge capacity is $723.8 \mathrm{mAh} / \mathrm{g}$; the discharge capacity is $600 \mathrm{mAh} / \mathrm{g}$ after 100 cycles at $100 \mathrm{~mA} / \mathrm{g}$ 


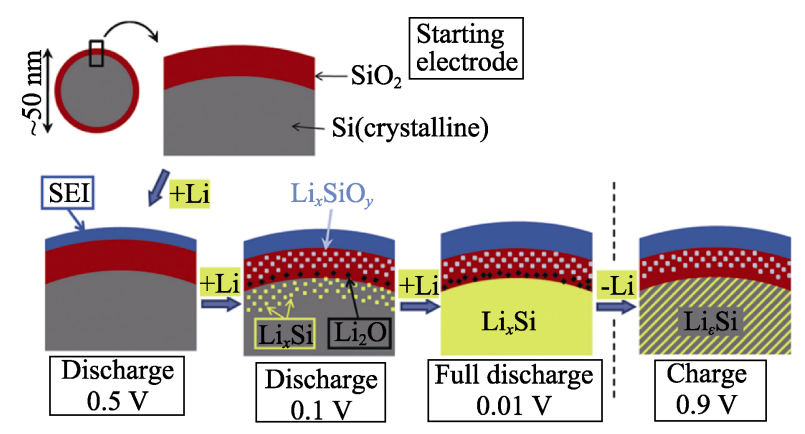

图 $9 \mathrm{SiO}_{x}$ 材料的基本电化学机理示意图 ${ }^{[46]}$

Fig. 9 Schematic diagram of the basic electrochemical mechanism of $\mathrm{SiO}_{x}$-based materials ${ }^{[46]}$

涂覆一层锂粉, 再放入电解液中浸泡进行预锂化强 化处理, 使得 $\mathrm{Li}$ 与 $\mathrm{SiO}$ 形成 $\mathrm{Li}_{4} \mathrm{SiO}_{4}$ 相, 其电极的 首次库仑效率为 $73 \%$ 。Kim 等 ${ }^{[51]}$ 开发了一种可扩展 卷绕的新型受控预锂化方法，原始电极通过金属锂
箔的电路短路实现预锂化, 如图 10 所示, 同时可以 监测两个电极之间的电压, 预锂化处理的 $\mathrm{SiO}_{x} / \mathrm{C}$ 复 合材料首次库伦效率高达 $94.9 \%$ 。

\subsubsection{3 多孔化设计}

复合材料的多孔化设计有利于锂离子在孔道中 快速传输, 有助于提高电极材料的倍率性能 ${ }^{[52-56]}$ 。Yu 等 ${ }^{[33]}$ 对歧化处理的 $\mathrm{SiO}$ 材料, 用 $\mathrm{NaOH}$ 溶液腐蚀去 除氧化硅基体中的部分纳米 $\mathrm{Si}$ 颗粒，最终制得了多 孔 $\mathrm{Si} / \mathrm{SiO}_{x}$ 材料, 该材料循环 100 次后容量保持在 $1242 \mathrm{mAh} / \mathrm{g}$ 。Feng 等 ${ }^{[54]}$ 通过镁热还原法制备的多孔 $\mathrm{SiO}_{x} / \mathrm{Si} / \mathrm{C}$ 复合材料(图 11)所示, 该材料可逆容量为 $1250 \mathrm{mAh} / \mathrm{g}$, 经过 100 次循环后容量保持率为 $90.9 \%$, 具有较好的倍率和循环性能。

此外, $\mathrm{SiO}_{x}-\mathrm{C}$ 复合材料受到多家日韩锂电企业 及研究所的广泛研究和青睐 ${ }^{[57-58]}$, 其中日本产业技

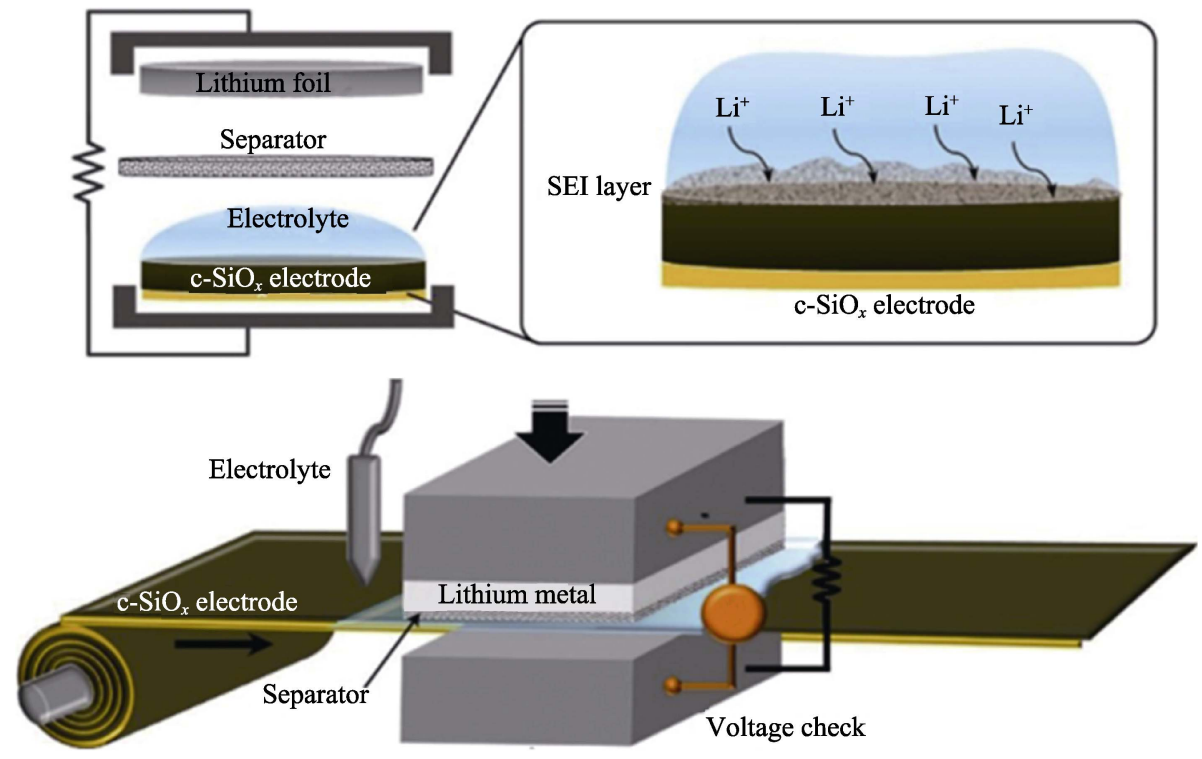

图 10 卷绕电池的金属管电路短路预锂化处理的示意图 ${ }^{[50]}$

Fig. 10 Schematic diagram of short-circuit prelithiation treatment of metal foil circuit of batteries ${ }^{[50]}$

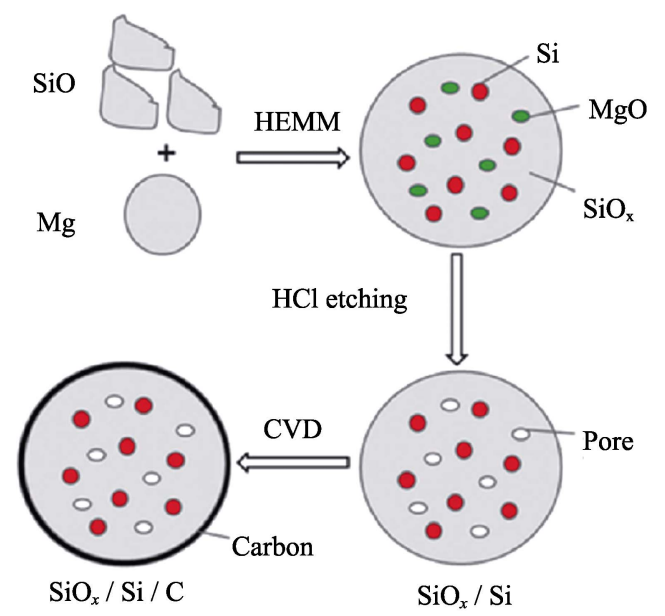

图 11 复合材料 $\mathrm{SiO}_{x} / \mathrm{Si} / \mathrm{C}$ 的制备示意图 ${ }^{[54]}$

Fig. 11 Schematic diagram of preparation of the $\mathrm{SiO}_{x} / \mathrm{Si} / \mathrm{C}^{[54]}$
术综合研究所将 $\mathrm{SiO} / \mathrm{C}$ 复合负极材料与磷酸铁锂正 极材料组装，该全电池循环 600 个周期后，容量保 持率达到 $90 \%$, 且表现出优异的倍率性能 ${ }^{[58]}$ 。国内 宁德时代新能能源公司 ${ }^{[59]}$ 制备的中空结构 $\mathrm{SiO} @$ void@C 复合材料(图 12)具有较优的电化学性能, 材 料的可逆比容量高达 $1305 \mathrm{mAh} / \mathrm{g}$, 可稳定循环长达 500 圈，容量保持率为 $54 \%$ 。

中国在“十三五”新能源汽车试点专项的共性关 键技术类研究项目“高比能量锂离子电池技术”中提 出: 到 2020 年, 电池单体能量密度 $\geqslant 300 \mathrm{Wh} / \mathrm{kg}$ 。 为此过去 20 年提出了多种技术解决手段, 目前商业 化复合材料的可逆容量在 $450 \mathrm{mAh} / \mathrm{g}$ 以下的碳包覆 氧化亚硅、碳包覆纳米硅碳复合材料在循环性、倍 


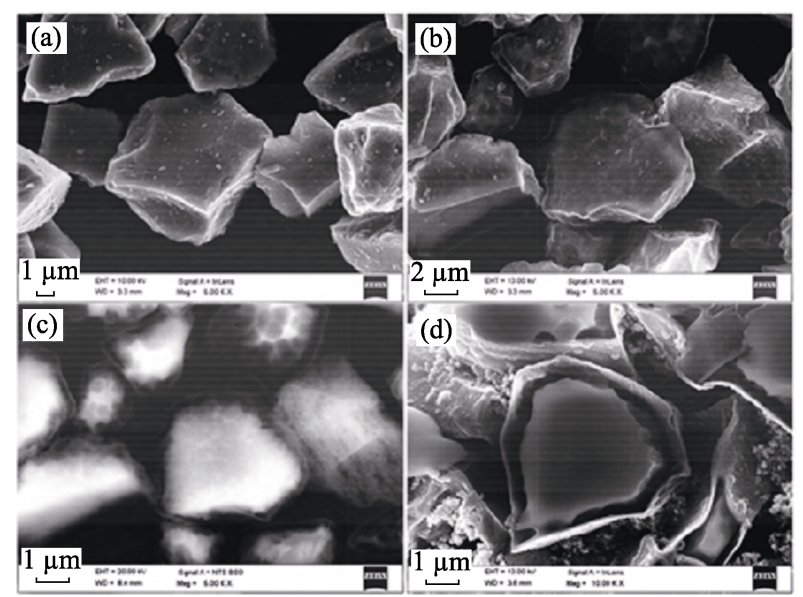

图 12 (a)初始 $\mathrm{SiO} @ \mathrm{C}$ 原料与中空 $\mathrm{SiO} @$ void@C 材料的表 面((b)二次电子相, (c)背散射相)及(d)截面 SEM 照片 ${ }^{[59]}$

Fig. 12 SEM images of (a) initial SiO@C, surface ((b) secondary electron phase, (c) back scattered) and (d) cross-section of hollowSiO@void@C material ${ }^{[59]}$

率特性方面基本能满足应用要求，已开始小批量进 入电动工具等市场。但 $450 \mathrm{mAh} / \mathrm{g}$ 以上的硅基负极 材料应用还有很多技术挑战。综上，在硅基复合材 料维度及结构设计时, 应更加着重于提高硅基负极 材料选择和制备的性价比，无需片面追求硅基负极 复合材料过高的比容量, 当性价比较高的硅基复合 材料的比容量能在 $750 \mathrm{mAh} / \mathrm{g}$ 左右稳定循环 500 次 以上, 即可有效提高电池的能量密度, 实现 2020 年 $\geqslant 300 \mathrm{Wh} / \mathrm{kg}$ 目标。

\section{3 挑战和展望}

未来高比容量硅基材料选择和结构设计的两个 主要发展方向是硅碳复合材料和 $\mathrm{SiO}_{x}(0<x \leqslant 2)$ 基复 合材料, 但是二者在本质机理上都存在严重的体积 效应。从几种典型硅基负极材料性能对比图(图 13) 可知, 为了克服体积膨胀问题并加快商业化应用进 程，需要从以下几个方面解决:

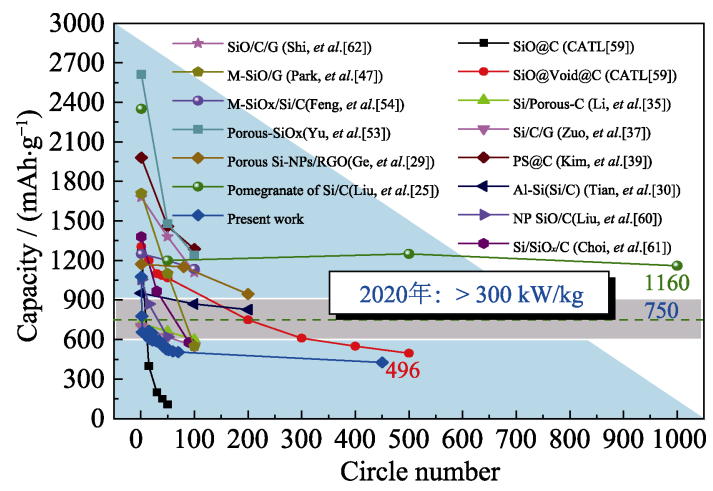

图 13 几种典型硅基负极材料性能对比 ${ }^{[25,30,35-39,47,53-54,59-62]}$

Fig. 13 Performance comparisons of common Si-based anode materials $^{[25,30,35-39,47,53-54,59-62]}$
1) 通过特殊制备方法得到具有特殊结构且性 能非常优异的硅碳复合负极材料, 对科研发展具有 重要的指导意义, 但是很多研究成果的制备流程冗 长且成本较高, 很难短时间内实现产业化生产, 因 此寻求成本更低、更易产业化生产的方法, 是高比 能量硅基电极材料产业化亟需解决的问题。同时, 考虑到正负极材料的容量匹配问题，硅基复合材料 只要能稳定循环 500 个周期以上，比容量还保持在 $750 \mathrm{mAh} / \mathrm{g}$ 左右, 仍可有效提高锂离子电池的能量 密度。

2) 相比 $\mathrm{Si}$ 材料, $\mathrm{SiO}_{x}$ 材料的体积膨胀效应更小, 是短期可实现商业化最有潜力的选择。有效地制备 出循环性能和倍率性能更加优越的 $\mathrm{SiO}_{x} / \mathrm{C}$ 复合材料 仍将是日后研究的重点。同时需要进一步系统研究 $\mathrm{SiO}_{x}$ 材料的预锂化和电化学机理。

3) 硅基材料的导电性较差, 故在硅基材料中 引入金属元素, 设计提高硅基材料的导电性和循 环性能的硅/金属复合材料结构也将是今后一个研 究热点。

\section{参考文献:}

[1] GOODENOUGH J B, PARK K S. The Li-ion rechargeable battery: a perspective. J. Am. Chem. Soc., 2013, 135(4): 1167-1176.

[2] TAN Y, XUE B. Research progress on lithium titanate as anode material in lithium-ion battery. J. Inorg. Mater., 2018, 33(5): 475-482.

[3] LUO W, CHEN X, XIA Y, et al. Surface and interface engineering of silicon-based anode materials for lithium-ion batteries. Adv. Energy Mater., 2017, 7(24): 1701083-1-28.

[4] XIAO Q Z, FAN Y, WANG X H, et al. A multilayer Si/CNT coaxial nano fiber LiB anode with a high areal capacity. Energy Environ. Sci., 2014, 7(2): 655-661.

[5] HUANG S, FAN F, LI J, et al. Stress generation during lithiation of high-capacity electrode particles in lithium ion batteries. Acta Mater., 2013, 61(12): 4354-4364.

[6] LI J, DAHN J R. An in situ X-ray diffraction study of the reaction of Li with crystalline Si. J. Electrochem. Soc., 2007, 154(3): A156-A161.

[7] WANG F, WU L J, KEY B, et al. Electrochemical reaction of lithium with nanostructure silicon anodes: a study by in-situ synchrotron X-ray diffraction and electron energy-loss spectroscopy. Adv. Energy Mater., 2013, 3(10): 1324-1331.

[8] OBROVAC M N, KRAUSE L J. Reversible cycling of crystalline silicon powder. J. Electrochem. Soc., 2007, 154(2): A103-A108.

[9] DING N, XU J, YAO Y X, et al. Improvement of cyclability of Si as anode for Li-ion batteries. J. Power Sources, 2009, 192(2): 644-651.

[10] SETHURAMAN V A, CHON M J, SHIMSHAK M, et al. In situ, measurements of stress evolution in silicon thin films during electrochemical lithiation and delithiation. J. Power Sources, 2012, 195(15): 5062-5066.

[11] NADIMPALLI S P V, SETHURAMAN V A, BUCCI G, et al. On plastic deformation and fracture in Si films during electrochemical lithiation/delithiation cycling. J. Electrochem. Soc., 2013, 160(10): A1885-A1893. 
[12] GHASSEMI H, MING A, CHEN N, et al. In situ electrochemical lithiation/delithiation observation of individual amorphous $\mathrm{Si}$ nanorods. ACS Nano, 2011, 5(10): 7805-7811.

[13] LIANG B, LIU Y, XU Y. Silicon-based materials as high capacity anodes for next generation lithium ion batteries. J. Power Sources, 2014, 267: 469-490.

[14] WEN Z S, WANG K, XIE J Y. Interface formed on high capacity silicon anode for lithium ion batteries. J. Inorg. Mater., 2007, 22(3): 437-441.

[15] CHAN C K, RUFFO R, HONG S, et al. Surface chemistry and morphology of the solid electrolyte interphase on silicon nanowire lithium-ion battery anodes. J. Power Sources, 2009, 189(2): $1132-1140$.

[16] KEY B, BHATTACHARYYA R, MORCRETTE $\mathrm{M}$, et al. Real-time NMR investigations of structural changes in silicon electrodes for lithium-ion batteries. J. Am. Chem. Soc., 2009, 131(26): 9239-9249.

[17] JI H R, KIM J W, SUNG Y E, et al. Failure modes of silicon powder negative electrode in lithium secondary batteries. Electrochem. Solid-State Lett., 2004, 7(10): A306-A309.

[18] HONG L, HUANG X, CHEN L, et al. The crystal structural evolution of nano-Si anode caused by lithium insertion and extraction at room temperature. Solid State Ionics, 2000, 135(1): 181-191.

[19] LIANG J W, LI X N, ZHU Y C, et al. Hydrothermal synthesis of nano-silicon from a silica sol and its use in lithium ion batteries. Nano Res., 2015, 8(5): 1497-1504.

[20] KIM W S, CHOI J, HONG S H. Meso-porous silicon-coated carbon nanotube as an anode for lithium-ion battery. Nano Lett., 2016, 9(7): 2174-2181.

[21] ZHOU Y N, XUE M Z, FU Z W. Nanostructured thin film electrodes for lithium storage and all-solid-state thin-film lithium batteries. J. Power Sources, 2013, 234(21): 310-332.

[22] DATTA M K, MARANCHI J, CHUNG S J, et al. Amorphous siliconcarbon based nano-scale thin film anode materials for lithium ion batteries. Electrochim. Acta, 2011, 56(13): 4717-4723.

[23] CHENG H, XIAO R, BIAN H, et al. Periodic porous silicon thin films with interconnected channels as durable anode materials for lithium ion batteries. Mater. Chem. Phys., 2014, 144(1/2): 25-30.

[24] TONG Y, XU Z, LIU C, et al. Magnetic sputtered amorphous Si/C multilayer thin films as anode materials for lithium ion batteries. $J$. Power Sources, 2014, 247(2): 78-83.

[25] LIU N, LU Z, ZHAO J, et al. A pomegranate-inspired nanoscale design for large-volume-change lithium battery anodes. Nat. Nanotechnol., 2014, 9(3): 187-192.

[26] XIE J, TONG L, SU L, et al. Core-shell yolk-shell Si@C@Void@C nanohybrids as advanced lithium ion battery anodes with good electronic conductivity and corrosion resistance. $J$. Power Sources, 2017, 342: 529-536.

[27] BANG B M, LEE J I, KIM H, et al. High-performance macro porous bulk silicon anodes synthesized by template-free chemical etching. Adv. Energy Mater., 2012, 2(7): 878-883.

[28] GE M, LU Y, ERCIUS P, et al. Large-scale fabrication, 3D tomography, and lithium-ion battery application of porous silicon. Nano Lett., 2014, 14(1): 261-268.

[29] GE M, RONG J, FANG X, et al. Scalable preparation of porous silicon nanoparticles and their application for lithium-ion battery anodes. Nano Res., 2013, 6: 174-181.

[30] TIAN H, TAN X, XIN F, et al. Micro-sized nano-porous Si/C anodes for lithium ion batteries. Nano Energy, 2015, 11: 490-499.

[31] LIU N, WU H, MCDOWELL M T, et al. A yolk-shell design for stabilized and scalable Li-ion battery alloy anodes. Nano Lett., 2012, 12(6): 3315-3321.
[32] KIM H, HAN B, CHOO J, et al. Three-dimensional porous silicon particles for use in high-performance lithium secondary batteries. Angew. Chem. Int. Ed., 2008, 47(52): 10151-10154.

[33] JIANG H, ZHOU X, LIU G, et al. Free-standing Si/graphene paper using Si nanoparticles synthesized by acid-etching Al-Si alloy powder for high-stability Li-ion battery anodes. Electrochim. Acta, 2016, 188: 777-784.

[34] WRODNIGG G H, WRODNIGG T M, BESENHARD J O, et al. Propylene sulfite as film-forming electrolyte additive in lithium ion batteries. Electrochem. Commun., 1999, 1(3/4): 148-150.

[35] LI M, HOU X, SHA Y, et al. Facile spray-drying/pyrolysis synthesis of core-shell structure graphite/silicon-porous carbon composite as a superior anode for Li-ion batteries. J. Power Sources, 2014, 248(2): 721-728.

[36] PAN Q, ZUO P, LOU S, et al. Micro-sized spherical silicon@ carbon@graphene prepared by spray drying as anode material for lithium-ion batteries. J. Alloys Compd., 2017, 723: 434-440.

[37] ZUO P, YIN G, MA Y, et al. Electrochemical stability of silicon/ carbon composite anode for lithium ion batteries. Electrochim. Acta, 2007, 52(15): 4878-4883.

[38] LI X, GU M, HU S, et al. Mesoporous silicon sponge as an antipulverization structure for high-performance lithium-ion battery anodes. Nature Commun., 2014, 5(5): 4105-1-7.

[39] KIM J S, HALIM M, BYUN D, et al. Amorphous carbon-coated prickle-like silicon of micro and nano hybrid anode materials for lithium-ion batteries. Solid State Ionics, 2014, 260: 36-42.

[40] MIN K K, BO Y J, JIN S L, et al. Microstructures and electrochemical performances of nano-sized $\mathrm{SiO}_{x}(1.18 \leqslant x \leqslant 1.83)$ as an anode material for a lithium(Li)-ion battery. J. Power Sources, 2013, 244: 115-121.

[41] TAKEZAWA H, IWAMOTO K, ITO S, et al. Electrochemical behaviors of nonstoichiometric silicon suboxides $\left(\mathrm{SiO}_{x}\right)$ film prepared by reactive evaporation for lithium rechargeable batteries. $J$. Power Sources, 2013, 244: 149-157.

[42] SCHULMEISTER K, MADER W. TEM investigation on the structure of amorphous silicon monoxide. J. Non-Cryst. Solids, 2003, 320(1): 143-150.

[43] HOHL A, WIEDER T, AKEN P A V, et al. An interface clusters mixture model for the structure of amorphous silicon monoxide (SiO). J. Non-Cryst. Solids, 2003, 320(1): 255-280.

[44] LÜ P P, ZHAO H L, WANG J, et al. Facile preparation and electrochemical properties of amorphous $\mathrm{SiO}_{2} / \mathrm{C}$ composite as anode material for lithium ion batteries. J. Power Sources, 2013, 237(259): 291-294.

[45] LIU X, ZHAO H L, JIE J Y, et al. $\mathrm{SiO}_{x}(0<x \leqslant 2)$ based anode materials for lithium-ion batteries. Prog. Chem., 2015, 27(4): 336-348.

[46] PHILIPPE B, DEDRYVÈRE R, ALLOUCHE J, et al. Nanosilicon electrodes for lithium-ion batteries: interfacial mechanisms studied by hard and soft X-ray photoelectron spectroscopy. Chem. Mater., 2017, 24(24): 1107-1115.

[47] PARK C M, CHOI W, HWA Y, et al. Characterizations and electrochemical behaviors of disproportionate $\mathrm{SiO}$ and its composite for rechargeable Li-ion batteries. J. Mater. Chem., 2010, 20(23): 4854-4860.

[48] MORITA T, TAKAMI N. Nano Si cluster- $\mathrm{SiO}_{x}-\mathrm{C}$ composite material as high-capacity anode material for rechargeable lithium batteries. J. Electrochem. Soc., 2006, 153(2): A425-A430.

[49] YANG X L, ZHANG P C, WEN Z Y, et al. High performance silicon/carbon composite prepared by in situ carbon-thermal reduction for lithium ion batteries. J. Alloys Compd., 2010, 496(1): 403-406.

[50] SEONG I W, KIM K T, YOON W Y, et al. Electrochemical be- 
havior of a lithium-pre-doped carbon-coated silicon monoxide anode cell. J. Power Sources, 2009, 189(1): 511-514.

[51] KIM H J, CHOI S, LEE S J, et al. Controlled prelithiation of silicon monoxide for high performance lithium-ion rechargeable full cells. Nano Lett., 2016, 16(1): 282-288.

[52] XING A, ZHANG J, BAO Z, et al. A magnesiothermic reaction process for the scalable production of mesoporous silicon for rechargeable lithium batteries. Chem. Commun., 2013, 49(60): 6743-6745.

[53] YU B C, HWA Y, KIM J H, et al. A new approach to synthesis of porous $\mathrm{SiO}_{x}$, anode for Li-ion batteries via chemical etching of $\mathrm{Si}$ crystallites. Electrochim. Acta, 2014, 117(4): 426-430.

[54] FENG X J, YANG J, LU Q W, et al. Facile approach to $\mathrm{SiO}_{x} / \mathrm{Si} / \mathrm{C}$ composite anode material from bulk $\mathrm{SiO}$ for lithium ion batteries. Phys. Chem. Chem. Phys., 2013, 15(34): 14420-144206.

[55] YANG T, XIAO L I, TIAN X D, et al. Preparation and electrochemical performance of $\mathrm{Si} @ \mathrm{C} / \mathrm{SiO}_{x}$ as anode material for lithium-ion batteries. J. Inorg. Mater., 2017, 32(7): 699-704.

[56] LIU Y H, OKANO M, MUKAI T, et al. Improvement of thermal stability and safety of lithium ion battery using $\mathrm{SiO}$ anode material. J. Power Sources, 2016, 304: 9-14.
[57] MIYUKI T, OKUYAMA Y, SAKAMOTO T, et al. Characterization of heat treated $\mathrm{SiO}$ powder and development of a $\mathrm{LiFePO}_{4} /$ $\mathrm{SiO}$ lithium ion battery with high-rate capability and thermo stability. Electrochemistry, 2012, 80(6): 401-404.

[58] MASAYUKI Y, KAZUTAKA U, ATSUSHI U. Performance of the "SiO"-carbon composite-negative electrodes for high-capacity lithium-ion batteries; prototype 14500 batteries. J. Power Sources, 2013, 225: 221-225.

[59] LIU X. Facile synthesis and electrochemical performance of hollowSiO@void@C composite as anode material for lithium-ion batteries. Chin. Batt. Indust., 2017, 21(6): 3-9.

[60] LIU W R, YEN Y C, WU H C, et al. Nano-porous SiO/carbon composite anode for lithium-ion batteries. J. Appl. Electrochem., 2009, 39(9): 1643-1649.

[61] CHOI I, MIN J L, OH S M, et al. Fading mechanisms of carboncoated and disproportionated $\mathrm{Si} / \mathrm{SiO}_{x}$ negative electrode $\left(\mathrm{Si} / \mathrm{SiO}_{x} / \mathrm{C}\right)$ in Li-ion secondary batteries: dynamics and component analysis by TEM. Electrochim. Acta, 2012, 85(1): 369-376.

[62] SHI C C, YAN X L, ZHANG L L, et al. High-performance $\mathrm{SiO} / \mathrm{C} / \mathrm{G}$ composite anode for lithium ion batteries. J. Inorg. Mater., 2013, 28(9): 943-948. 